\title{
Developing, Validating, and Obtaining Stakeholder Buy-In for Criteria for Applying Social Science to Policymaking
}

Authors (alphabetical): Alexander Etz, Fiona Fidler, Gary L. Klein, Rick Lempert, Arthur Lupia, Michelle N. Meyer, Amy O'Hara, Nancy Potok, Simine Vazire

\section{Introduction}

What we aim to do. We aim to present a roadmap for developing validated, objective criteria for establishing how much confidence decision makers should place in particular social and behavioral science research outputs. We identify many research challenges, and risks and opportunities involved with each.

The limits of current practice. Currently, judgements about whether social science evidence is decision- or policy-ready are unarticulated, subjective, non-transparent, and non-accountable. These judgements are important but difficult, especially given low success rates in large-scale replication studies and increased awareness that questionable research practices are often missed by peer reviewers and other gatekeepers. We therefore propose the development of criteria, or standards, for these judgements.

Although many criteria for promoting trustworthy science exist, they are often disciplineor purpose-specific, and we know little about how they overlap, match up, or differ across the various social sciences. Thus one research challenge is collating and synthesizing existing criteria, and identifying gaps in the way research ethos are taught and applied.

The existing SCORE program aims to develop confidence scores which indicate the likely reproducibility and replicability of research claims. What we are proposing goes beyond this, to address whether results are likely to be generalizable to, for example:

- alternative operationalizations of key variables,

- different populations

- $\quad$ other defensible analysis strategies.

Such assessments will help aid judgements about the application of research practice. The proposed criteria will also assessment of research quality, such as:

- whether a good research question has been posed

- appropriateness of the study design,

- soundness of proposed measures,

- justifiability of planned statistical inferences (e.g., only do confirmatory tests when appropriate, only draw causal inferences when appropriate, etc.).

- Where research falls short of ideal practice, which in the real world is often unavoidable, have the researchers provided other assurance that the conclusions are appropriately qualified.

As social science researchers increasingly seek to benefit from advances in data science technology and methods, new issues have arisen, particularly around the quality of the data on which study results are based. Data are today widely available from multiple sources, including open data on the web, private data sellers, and federal, state and local agencies of government. 
Data science advances allow researchers to more easily acquire these data and use them either alone or incorporated with other independently collected research data (quantitative and qualitative). The development of quality criteria becomes more complex in this environment.

Although widely accepted methods for measuring error and coverage in random sample surveys exist and can guide peer reviews and attempts at reproducing research results, we don't currently have standards for determining best practices when using or evaluating many types of combined data that are becoming more commonplace in research designs, such as data sets that combine statistical survey with commercial data (e.g., aggregated credit card data or aerial photographs), or data from government records (collected for program administration or delivery of services). The lack of standards leaves researchers, peer reviewers and those who seek to use research results for applied purposes without consistent without consistent guidance for assessing the quality and utility of data that underlie conclusions which a researcher may have drawn.

Hence one research need is to examine the different dimensions that must be assessed to evaluate quality when studies combined data sets. Outstanding questions include: issues related to transparency; fitness for different research purposes; privacy protections (e.g. ensuring against the re-identification of confidential data); the ownership of and access to combined data sets; biases that may affect combined data, perhaps as a result of the data combination; and dealing with the methodological complexities that may exist in processing combined data, particularly when combined data sets are large and the sources of the data are diverse. Research challenges associated with these questions include, but are by no means limited to:

- How to do we create and implement consistent documentation?

- How should quality ratings be developed and implemented?

- How do we balance the need for privacy against increased transparency and data access

- How do we develop consistent scientifically sound approaches for editing, imputation, weighting, and modeling of combined data?

These questions apply, of course, even when data sets are not large and data come from a single source, but they are more challenging as data sets become larger and multi-sourced. These questions must be addressed if policy advisors and decision-makers are to feel confident that social science research is providing evidence that is objective, reliable, relevant, and of high utility. As 'precedent-setting' methods and models increase, the need for widely accepted standards of quality and integrity will become more important.

Why this is new, and likely to succeed. Our proposal differs from some others that have been advanced in that it attempts to make judgements about the decision-readiness of evidence explicit and transparent. As mentioned, this goes beyond judgements about replicability and reproducibility (the domain of SCORE) to address not only questions of generalizability but also to suggest fundamental requisites of quality social science. Success is, of course not guaranteed, but the time is nonetheless propitious for advancing new standards. Policy makers have increasingly come to realize that the social sciences are indeed sciences capable of designing and implementing methodologically rigorous studies of the dimensions and causes of social problems and the effects of policies designed to address them. Moreover, there is an increasing realization that many problems that were at one time thought capable of purely technical solutions have at their heart a human component which the more technological sciences do not address. Problems of climate change are one example; no matter how effective technological innovations may be in helping the world address climate change, it seems likely that problems associated with science change cannot be adequately ameliorated unless human behavior also changes. These 
realizations are, for example, manifested in the investments that DARPA, IARPA and other government agencies have been making in the social and behavioral sciences. At the same time, reliance on social science solutions and studies have revealed the many ways in which social science research results are context dependent if not seriously flawed, and efforts are being made to both call attention to problems and to develop institutionalized means of addressing them such as the rethinking of statistical standards by professional organizations like the American Economics Association and the services provided by the Open Science initiative. Our standard setting suggestions are part of a larger effort to substitute collective judgments of what constitutes strong science for the more individual efforts of peer reviewers and journal editors which has been the primary quality control mechanism in the past. The time is ripe for this kind of effort.

What difference this will make. The ultimate goal of our effort is to improve evidence based decision making by improving the quality of the evidence that social scientists can offer both political and private sector actors. We recognize, however, that formalizing the criteria for decision-ready evidence, cannot be done in the abstract or even by knowledgeable consensus. Criteria themselves must be empirically testable to ensure they fulfil their intended function. Only by empirical testing can we begin to evaluate whether we are relying on the right cues to judge research. We must avoid an infinite regress, but it is nonetheless important to empirically validate those criteria that are seen as improving the quality and reliability of social science research. For example, the preregistration of study designs is thought to protect against data ransacking or other inappropriately goal oriented post hoc efforts to manipulate or present data so that it supports a desired conclusion. The success of this intervention might, for example, be tested randomly require some grantees but not others to preregistration their data collection and analysis plans and compare results in the two groups, ideally perhaps by replicating the results produced by the two groups to see if the results of pre-registered studies are more likely to replicate than the results of studies that were not pre-registered.

If we can construct and validate widely accepted criteria the scientific community will be better able to assess the validity of research, and decision makers developing policies or needing to take action will have a clearer idea of whether research results are of sufficiently high quality to justify basing consequential actions on them. In a federal government environment, the risk of making suboptimal decisions affecting billion dollar investments, workforce and training activities, public health, education, safety, and other critical matters will be reduced, perhaps substantially. The longstanding federal goal of evidence-based policies and initiatives will be far closer to realization.

Risks and costs of this endeavor. One major challenge is establishing evaluation metrics for proposed criteria. Evaluating whether developed criteria can sufficiently demarcate between high- and low-quality research, for example, will to some extent rest, at least initially on converging independent judgements about what high and low quality research is. But there is always a risk that a consensus may be wrong, or unachievable because of reasonable disagreements about how high and low quality research should be operationalized.

Even if valid criteria can be operationalized, there may be difficulties in implementation. To be usable the criteria must not only be relatively unambiguous and otherwise easy to apply, but they must be accepted and find widespread uptake in the relevant communities. Yet different 
research traditions may interpret the same requirements differently but there may be disciplinespecific objections to some of what universal criteria may require. There is also a risk that any criteria that can command a broad consensus will be too narrow and not capture enough of the research space, either giving too much low quality research a pass or categorizing too much research as "low confidence" or "not policy ready" because the criteria do not adequately capture the strengths of the research being evaluated.

Near-and long-term measures of success. In the near-term, one would want to check the ability to develop and implement criteria for high priority aspects of research that can be tested on pilot research projects with a variety of designs. These early assessments should test whether the criteria are scoped correctly, cover sufficient aspects of research, are relatively easy to implement, and can be monitored. Longer-term, a final set of criteria would be developed and implemented. The ongoing monitoring scheme could gather feedback for continuous improvement, identify barriers to implementation and address them, and identify how the overall research environment is affected by their implementation, such as effect on publishing research results. Monitoring might incorporate some of the machine learning approaches also being developed to assess quality of research.

\section{Developing and achieving acceptablevalidated criteriaforpolicy application of social science}

Three sets of criteria are needed to establish trustworthy social and behavioral science: criteria related to research transparency, criteria related to research quality, and criteria related to legal, ethical, and social issues.

\section{Transparency}

Criteria for transparency are relatively straightforward. Here we describe four types of transparency that scientific outputs can be scored on: Data and code transparency, materials transparency, design and analysis transparency, and conflict of interest transparency. These overlap substantially with some of the Transparency and Openness Promotion (TOP) guidelines (Nosek et al., 2015).

For computational research, a fully transparent scientific product should include the underlying data and code that was used to obtain the results. Moreover, the data and code should be FAIR (Findable, Accessible, Interoperable, Reusable: https://www.force11.org/group/fairgroup/fairprinciples). Some common challenges that get in the way of achieving transparency of data and code are poor commenting/meta-data, use of commercial software that is not accessible to all, failing to specify the versions of software that were used, or storage of data and code in a location that may not persist over time.

Another component of research transparency is for all relevant information necessary to recreate the study procedures to be available. Depending on the type of research, this may include experimenter scripts, questionnaires, stimuli, videos of the procedure, descriptions of the relevant contextual variables, descriptions of the specific reagents used, instructions to participants, etc. The goal of materials transparency is to make it easy for other researchers to replicate the study. 
A third component of research transparency is disclosing the timing of researchers' decisions when this timing is relevant for interpreting the evidence. For example, when conducting hypothesis tests (e.g., using p-values or Bayes Factors), or engaging in any statistical practice in which the interpretation of the statistic depends on how many tests were run (e.g., frequentist effect estimation), it is crucial to give readers a way to verify which tests (and how many tests) were planned. The most common way to do this is using pre-registration or a preanalysis plan, which is a time-stamped document outlining how data will be collected and analyzed. For pre-registered plans to fulfill this purpose, they must be specific enough to constrain "researcher degrees of freedom," that is, they must specify exact analyses and statistics that will be interpreted. Finally, researchers should be transparent about any potential conflicts of interest.

Quality

Research integrity includes several facets of quality. In addition to the quality of data collected as part of a research study, other important quality dimensions include (but are not limited to): construct validity (operationalization); internal validity; external validity; and statistical validity. As research methods evolve through more collaborative, open research, exploring similarly evolving concepts of quality will be necessary. Developing criteria that include traditional quality measures around research validity (described below) as well as recognizing that methodologies are changing, will require a multidisciplinary approach. More rigorous review may be needed for research results that are based on novel methods or that present challenges for interpretation.

\section{Construct Validity (Operationalization)}

Theories which drive scientific understandings of how things work are built on concepts and specify relations between them. Some social science concepts are relatively unambiguous and we have considerable, and at times excessive, confidence in the reliability of observations designed to measure them. Thus, we take self-assessments of gender, often indicated by a check mark in a box, as a reliable indicator of the gender of the person who checked the box. Other social science concepts are, however, not so straightforward or easy to measure, and the degree to which certain observations measure them may be contested and of questionable reliability. Stress and discrimination are examples.

Empirical social science cannot work directly with theoretical concepts but can only work with indicators it can observe and measure. These indicators are operational definitions of the concepts they are taken to represent. Cortisol levels, for example, may be taken as indicators of levels of stress, or the proportion of businesses that invite a white job applicant in for a job interview as compared to the proportion of businesses in a job sector that invite a black applicant for a job interview when the applications of the two are, except for indicators of race, identical may be taken as a measure of job sector discrimination. Accepting these operational definitions, elevated cortisol levels when a person is asked to give a brief speech against her values may be taken as an indicator that arguing against one's values is stressful, and finding that businesses in one sector of the economy, restaurant workers for example, show more biased job interview invitations than another, short haul moving for example, may be taken to mean that discrimination against blacks is greater in the restaurant industry than in short haul moving. 
It is, however, easy to be misled. An operationalization may be valid (let's assume that cortisol levels are a valid measure of stress) but the presumed cause of changes in that variable may be wrong, calling into question the theory that predicted the relationship. For example, the elevated cortisol level of a person asked to give an argument that runs counter to her values may be due to the fact that she must get up to speak to a group rather than to what she is being told to say. Or the operationalization may itself be questionable or be taken to mean more than it in fact does. For example, it may, hypothetically, be true that a black applicant is relative to white applicants more likely to secure a job interview from a short haul trucking company than from a restaurant, but the black workers for the trucking company may be paid less than their white counterparts for the same work and be treated more hostilely by their co-workers while those blacks who are hired by restaurants may receive the same wages and privileges as their white counterparts and may report encountering no race-based hostility on their jobs. So, in which sector is discrimination more rampant? Using one defensible measure of discrimination, it is the restaurant industry because their hiring processes are influenced by applicant race. Using another measure, it is the short haul trucking industry because blacks who work in this industry are treated worse than whites when the same is not true of blacks who work in the restaurant industry.

Only by paying close attention to how a concept is operationalized can we understand the relationships we have discovered, but the temptation is too often to create an operational definition, attach it to a concept that is generally speaking less technically and more broadly understood not just in the larger community but sometimes in scientific communities as well, and then treat the operationalized measure as if it is in fact what is meant when we use the concept it is designed to operationalize. The classic example is IQ, which is understood by many not just as a measure of intelligence but as a synonym for it. Yet reported IQs are only scores on a test. They may measure some kinds of intelligence (e.g., the intelligence needed to get good grades in many college courses) reasonably well but may do a poor job of measuring other kinds of intelligence (e.g., the intelligence needed to get along well in groups.) Moreover, to the extent IQ is taken to measure some inner capacity for solving certain kinds of problems it may systematically mislead because the items used to measure it may be culturally biased or be testing English language proficiency more than they are a capacity for learning or problem solving. Both theory and policy may suffer if insufficient attention is paid to how the concepts that drive them have been operationalized in those empirical studies that are designed to shed light on the likely truth of different hypotheses and the likely effects of particular policies.

Some operational definitions are, to be sure, better than others in the sense that they are more valid; i.e., some bear a closer relationship to the concepts they are intended to measure than others. Indeed, our ability to generalize from the results of social science (and indeed all science) research depends on construct validity: the validity of the operational definitions that we use both to generate theoretical propositions to be tested and to test propositions that for either inductive or logical/mathematical reasons are thought plausible. In some instances, we may be satisfied with face validity because we have little reas on to doubt the validity of a measure or are confident in the process used to generate it. For example, some people may accidentally or intentionally check the wrong box on a form when asked to indicate their gender, but in most settings if we simply add up the number of people who checked male and who checked female on a questionnaire even though we may not capture the exact split of respondents by gender we are likely to come very close. Hence, there is little reason to suspect that these lies or mistakes will be so common as to substantially undercut the validity of differences reported by gender. 
Similarly, we know that errors may be made in determining age-adjusted mortality rates, but knowing the careful process used to generate these measures, the unambiguity of death and the likely reliability of ages attributed to deceased individuals, we have considerable reason to believe the figures are relatively accurate, with any errors distributed at random in ways that are unlikely to distort most findings that use age-related mortality as a variable.

Often, however, the validity of operational definitions and limits on that validity must be demonstrated empirically. One way to do this is by reference to a criterion that we see as a more direct measure of the concept. For example, Law School Aptitude Scores (LSAT scores) are supposedly a measure of the likelihood that one will do well in law school. A more direct measure of how well a person does in law school is the grades that a person receives. When we compare within schools the LSAT scores that students have achieved with the grades they get, we see that the measure has some degree of validity since there is a reasonably strong association between LSAT scores and law school grades, at least in the first year. The association is, however, far from perfect and the fact that one student has a higher LSAT score than another does not mean that the higher scoring student will do better in her law school classes. Also, we must be careful that we do not, without more, treat LSAT scores as measures of a person's aptitude for success in the practice of law. No study validates LSAT scores as indicators of practice success. Treating them as practice success indicators rests on assumptions and not empirical social science. Yet an argument sometimes made against affirmative action is that because its beneficiaries have lower LSAT, MCAT or other admissions test scores than their white classmates, a result of affirmative action will be to produce incompetent lawyers. The few empirical studies that have been done refute this claim.

Another way of validating operational definitions is by checking in a variety of context using a variety of methods whether the operationalized measure behaves as we would expect it would if it in fact measured the concept it is claimed to represent. For example, if cortisol levels rise when a people expect to receive an electric shocks, or are told they have failed an important test, or have insufficient time to finish an assignment, or are told they must reveal an embarrassing fact about themselves and does not rise or falls when they are praised or told they will soon be allowed to choose a gift from a prize bag, we have good reason to believe that cortisol is a valid operationalization of being stressed. This is because we have good reason to think that the situations used for test purposes are likely to be stressful or not, and cortisol levels change in the ways we would expect them to if they rose in response to stress and were stable or dropped when situations are not stressful.

I short, in doing and reviewing empirical research operational definitions of key variables demand careful attention. Strictly speaking, the data we collect and relationships we find relate only to our measures as operationalized. We may, however, have good reason to believe that our operationalized variables reflect concepts broader than their precise narrow scopes. This, however, is an empirical question and to the extent possible should be empirically validated. We may also have sound reasons for assuming that a particular operationalization is conceptually even broader than what has been validated empirically, but we should be aware that at this point the meaning we attribute to a measured variable begins to rest on theory and assumptions rather than on empirical social science, and we should be aware and acknowledge other plausible meanings that the variables we measure may have. 


\section{Internal Validity}

Internal validity refers to the validity of causal claims. There are many threats to internal validity in both experimental and correlational research. In experimental research, threats to internal validity include: confounds, demand characteristics, failures of random assignment, and poor experimental control. In correlational research, causal claims are less common but can be justified under some very specific and rare conditions (Rohrer, 2018).

\section{External Validity}

External validity refers to the validity of claims made about the generalizability of a finding. For example, researchers sometimes claim that the observed effect would generalize to other populations, settings, time periods, cultures, species or operationalizations. Many scientific claims are only interesting if these claims about generalizability are valid. Two types of external validity include population and ecological validity. Population validity asks whether the sample population used in the research represents the entire population. Often, the answer to this question is sought by looking at the methods used to select the sample; i.e., whether the sample was selected randomly or was a convenience sample, and whether the sample was representative of the population as a whole. Ecological validity assesses whether the testing environment itself had an influence on the results. If the environment of the test doesn't resemble real world conditions, the results achieved in the test environment may not be generalizable outside of the that environment.

\section{Statistical Validity}

Statistical validity refers to the validity of statistical inferences made from data. In the social sciences, it is common to use frequentist statistics and hypothesis testing approaches. For example, Null Hypothesis Significance Testing (i.e., comparing a p-value to the alpha threshold to determine statistical significance) is common in many areas of social science. There are many common threats to statistical validity, including flexibility in data collection and analysis that can be used (intentionally or not) to capitalize on chance, which increases the rate of false positives. Cherry-picking results (e.g., failing to report negative results) or changing one's hypothesis after seeing the results are also threats to valid statistical inference.

\section{Research Challenges Related to Transparency and Quality Criteria}

Here we summarize challenges related to our proposal so far.

1. Aggregating and synthesizing existing criteria. Meeting this challenge involves answer questions such as: What is the interplay between the different existing standards? Can they be combined and/or weighted. Where are the gaps? How useable are current standards? What are the challenges to uptake?

2. Evaluating whether new criteria/standards discriminate between high and low quality research, and whether adhering to them improves judgements about the decisionreadiness/trustworthiness/integrity of research? 
3. Avoiding mindless application of standards. When should criteria be loosened/exceptions made? When are flawed data still useful?

4. Developing custom standards. What are the distinct kinds of data that each need their own standards (e.g., combined data, survey data, experimenal data, $\mathrm{N}=1$ designs, etc.)? Will it be possible to develop standards for the large proportion of social science research that is exploratory? Is exploratory research less amenable to metrics?

5.Implementing and maintaining integrity of standards. We have outlined challenges of acceptance and uptake. Further questions here include what can be done about gaming of metrics? How do we ensure ongoing evaluation and improvement of standards?

\section{Legal, ethical, and social considerations: Research Challenges and Opportunities}

In addition to having confidence that social science research upon which they may rely in shaping policy is sufficiently transparent and of sufficiently high quality, policy makers should have confidence in the legal and ethical status of the underlying social science research and in the legal and ethical appropriateness of applying social science research to a particular policy. (Although we here separately address certain legal and ethical issues, we note that ensuring that research on which policy relies is transparent and of high scientific quality are themselves ethical issues of the utmost importance.)

Challenge: Uncertain legal and ethical standards governing some contemporary social science research. All research involving human participants requires ethical-legal consideration. Ordinarily, established bodies — such as institutional review boards (IRBs), funders, and journals - prospectively and retrospectively apply relatively clear regulatory and ethical frameworks in determining whether research will be, or was, conducted responsibly. However, contemporary and emerging social science methods sometimes test the limits of those familiar frameworks - if those frameworks apply at all. Under the U.S. federal Common Rule, for instance, which has been largely unchanged since 1991, much depends on whether data are "identifiable" or not. Neither those regulations nor federal administrative guidance interpreting "identifiability" reflects relatively recent developments in re-identification techniques made possible by the ever-increasing amount of data that is available about each of us. After six years of debate, the Common Rule was revised to require agencies periodically to reconsider the definition of "identifiability," as well as whether some techniques inherently yield identifiable data - but this merely prolongs the uncertainty.

Similarly, much depends under the Common Rule on whether data are "publicly available." But relatively new platforms that have emerged since the early 1990s do not involve plainly public or private data.

The Common Rule provides for waiver or alteration of informed consent when, inter alia, "the research could not practicably be carried out" without it. Does the scale and speed at which some social science now occurs (e.g., behavioral A/B testing on social media platforms) render it "impractical" to obtain consent, or at least study-specific consent? Is broad consent to research (say, at the point of establishing an account or other presence on a platform) an appropriate 
substitute for study-specific consent, and if so, how detailed must such consent be about the possible research uses of data?

Finally, the Common Rule only applies to studies that are federally funded or conducted at an institution (such as most universities) that have committed to applying the Common Rule to all of its human subjects research, regardless of funding. Increasingly, however, important social science research is conducted by industry or by academic-industry partnerships (where the academic institution may not be "engaged in research," and so not subject to the Common Rule).

The gaps in the coverage, and uncertainties about proper interpretation, of the Common Rule shift more weight on ethical standards governing contemporary social science, especially as it leverages big data. But no such agreed-upon standards currently exist. For instance: When are data "public" and what follows from designation of data as public? Can risks of re-identification be reliably assessed, and how much risk of re-identification is too much? Are prospective group review and specific informed consent ethically required before data collected for one purpose (e.g., administrative) are shared and/or analyzed for other purposes? As a result of the foregoing, policy-makers may lack confidence in the ethical and/or legal status of some research, even when that research has passed ethical-legal evaluations by IRBs, funders, and/or journals.

Opportunities: Convene major industry actors who play important roles in the data ecosystem in order to develop a consensus about the voluntary adoption of ethical standards for responsible data use. In the absence of useful guidance from OHRP or other federal regulators, convene experts in research ethics and regulation to develop guidance, specific to social science research, addressing the above questions (including social-science-specific interpretations of the Common Rule and ethical standard applicable when the Common Rule does not apply).

Challenge: Retrospective application of new ethical-legal standards. Assuming that consensus is reached on appropriate legal and ethical standards for conducting social science research, a great deal of social science research will, of course, have been conducted before that consensus was reached. When is it ethically acceptable for policy-makers to rely on research that may not have met current ethical and/or legal standards?

Challenge: Inappropriate policy uses of social science. Even if there is reasonable expert consensus that social science research was ethically and legally conducted (as judged by the standards of the time or by current standards), it may not be ethical to apply that research to a particular policy context. For instance, even if facial recognition was extremely accurate, and even if the research, it might still be wrong for a policy-maker to use it in certain public settings.

Challenge: Even if there is reasonable expert consensus that social science research was ethically and legally conducted and that it would be ethical to apply it to a particular policy context, the general public may reject either the underlying research and/or the application of social science to policy. Similarly, where consent to social science research and/or data sharing is sought, uptake may be low owing to problematic perceptions of "research," "experimentation," and data sharing. For example, there is evidence that the general public (and even experts) are less comfortable with a randomized evaluation of an untested policy in half the population than with simply implementing it, untested, in the entire population. Similarly, although some "nudges" are well tolerated, some are not — and not always for obvious reasons (i.e., reasons that classical economics would deem "rational"). Public disapproval of these social science methods and interventions may prevent policy-makers from relying on them, even if the evidence suggests that they would improve welfare or other outcomes of interest.

Opportunities: More research is needed investigating why many people disapprove of critically important activities such as randomized evaluation, behaviorally-informed policy, and 
data sharing, and how these activities can be messaged to increase acceptance of these social science methods and interventions.

Challenge: A great deal of social science research continues to be based on WEIRD populations. Results of such research may not generalize to other populations, who will, by contrast, ordinarily be subject to policies that rely on this research.

Challenge: Because social scientists overwhelmingly identify as politically left-leaning, the vast majority of social science research is produced by left-leaning scientists. This has contributed to skepticism among policymakers that social science research is credible.

Opportunity: Fund "adversarial collaborations" among researchers of different ideological persuasions to conduct or replicate policy-relevant research.

Challenge: Above, we have called for transparency in research data that would be used in policymaking, including raw data, where possible. However, re-identification poses a threat to ethically (and, in some instances, legally) acceptable data sharing. A fair amount of attention has now been paid to the technological capacity for re-identification of various kinds of data, but relatively little attention has been paid to the likelihood of re-identification attacks, given attacker incentives and the effort required under various scenarios.

Opportunities: Examples of activities:

- Further research applying game theory to various re-identification threats

- Once re-identification risk is better (albeit imperfectly) quantified, the public's preferences for data sharing under risk versus limited or no data sharing should be rigorously investigated (perhaps using discrete choice methods).

- Development of automated processes for assessing re-identification risk of particular datasets and for de-identifying them and/or producing synthetic datasets to be used instead.

- Exploration of social safety net where victims of re-identification are compensated (cf. National Vaccine Injury Compensation Fund).

- Honest brokers

Challenge: A policymaker in one jurisdiction may overlook research integrity challenges that are unique to the jurisdiction in which the research was conducted and may therefore incorrectly conclude that there are no social, ethical or legal issues with the underlying research.

\section{Getting stakeholders to accept the criteria}

Challenge: How do we talk about standards in a way that is understandable to a broad audience? (Can we train decision-makers to better understand uncertainty/complexity?)

Challenge: If our standards/criteria are counterintuitive to decision-makers/the public, what evidence will we use to convince decision-makers that our standards are valid? How can they test our standards against their own preferred standards?

Challenge: Many standards already exist (e.g., OMB, NIST, UN) and people ignore them. How do we get people to pay attention \& use standards? This is a culture change problem. Who are the people we need to convince?

Challenge: What are the incentives that are in the way of researchers adopting high standards? 
Challenge: Is there some "seal of approval" that would help communicate to the public which findings meet the highest standards? Currently people use acceptance for publication as this kind of seal - we would need to avoid all the problems that this has revealed.

Many individuals, institutions, businesses, and researchers want to use scientific insights to more effectively or efficiently achieve important aspirations. Scientific findings, however, are often conveyed in ways that are difficult for non-specialists to understand. Hence, many scientific organizations have devoted increasing effort in recent years to improving science communication.

Given a particular level of communicative effectiveness, the question then becomes - Of what value is the information that is being conveyed? Potential users of a research finding usually have a particular domain of application in mind. To learn about whether a specific finding applies to a particular domain, potential users need to learn about the context in which the scientific finding was produced.

Scientific research varies in the amount of contextual detail that it makes available. Some research offers significant detail about how researchers chose phenomena to observe and how they chose categorical, analytic, and interpretive frameworks for characterizing their observations. These choices, in turn, affect the types of existential, correlational, and causal conjectures that can be evaluated.

To make effective choices about whether and how to apply a research finding, potential users will employ a standard. The standard will influence, or perhaps, determine, these choices. This section addresses questions about types of standards that can help prospective users make more effective use of scientific findings.

For standards to have this effect, the standards should place significant weight on attributes of research that correspond to reliable and accurate inferences. If a prospective user imagines using the standard to produce comparative evaluations of claims made within large academic literatures, then the standard will be efficient and effective only if the attributes of research weighted more heavily in the standard correspond to research practices that many scholars are willing and able to use. If an end user chooses a standard that in no way corresponds to scholarly practice, then they will lose the ability to derive value from the associated literatures.

Are there scholarly standards that are widely adopted and that correspond to greater levels of reliability for prospective end users?

A challenge is that for any scholarly domain, an infinite number of standards can be produced. Many scholarly literatures examine complex problems. One can characterize such problem spaces as having a large number of dimensions. Each dimension can be given a wide range of relative weights. Dimensions can also be combined or compressed for evaluative purposes. An infinite number of combinations can be used to develop standards. Moreover, an infinitely-sized subset of standards have potentially desirable attributes. So choices must be made.

Wide scholarly adoption requires broad perception that a standard is legitimate. A legitimacy claim itself requires a comparison. It requires a belief that operating under the proposed standard will produce better outcomes than affected groups of scholars have now.

It is important to realize that all people already operate with respect to standards. They may vary in their ability to articulate the standard, but everyone has standards in mind that guide their decisions and evaluations. 
The question then turns to perceptions. Why would someone replace their existing standard with a new standard? A requirement for replacement is incentive-compatibility. A person must believe that switching standards will have beneficial effects. Such beliefs, however, will be grounded in past practices. Hence, all else constant, scholars are likely to resist new standards unless they are associated with iconic successes that ambitious scholars have incentives to emulate.

A challenge at such circumstances is motivated reasoning. People tend to favor evaluate standards that elevate attributes that they already see as desirable. Think, for example, about rankings of academic departments. These rankings are evaluated with respect to a standard. Different rankings come from different standards. Many reactions to these standards are based not on methodological concerns but on whether universities for which the viewer has an affinity do better (or worse) than expected with respect to the ranking. People whose affinity institutions do better than expected are more likely to see a given standard as valuable than people whose affinity institutions do worse than expected.

Hence, a requirement for wide adoption of a new standard requires that the standard have an explicit tie to aspirational high-value outcomes. Prospective adopters must be convinced that hewing to the standard offers a more effective or efficient way to achieve already existing goals.

Consider, for example, the evolving acceptance of Google Scholar (GS). Before GS, many researchers relied on ISI indices to count citations. Google and ISI use different counting standards. GS's standard produces higher citation counts. This attribute of GS is particularly important for younger scholars who have strong professional incentives to demonstrate impact. I do not know whether ISI or GS is a more valid estimate of scholarly impact. I do know that GS produces larger numbers for more scholars. For that reason, and all else constant, it is more likely to gain adoption.

If it were true that GS is better at counting activities that correspond to high-value outcomes, then wide adoption of that standard would be beneficial to the mission of agencies that value those outcomes. If it were true that ISI was better at counting such activities, then wide adoption of GS would be harmful.

Therefore, the value of a scientific standard to a mission agency depends on wide adoption and a true link from measured activities to high-value outcomes. Since wide adoption depends on perceptions of legitimacy and since perceptions of legitimacy require incentive compatibility, value-adding standards require both:

- a true link to the mission agency's high-value outcomes, and

- prospective adopters to perceive higher scores on these metrics as consistent with preexisting aspirations.

\section{Conclusion}

